\title{
Vibrational Analysis of Levy-Type Plates by Using SEM
}

\author{
Shota Kiryu ${ }^{1}$ and Buntara Sthenly Gan ${ }^{2 *}$ \\ ${ }^{1}$ Student, Departement of Architecture, Graduate School of Engineering, Nihon University, \\ 1 Nakagawara, Tokusada, Koriyama, Fukushima 963-8642, Japan \\ ${ }^{2}$ Professor, Departement of Architecture, College of Engineering, Nihon University, \\ 1 Nakagawara, Tokusada, Koriyama, Fukushima 963-8643 \\ * Corresponding author:buntara@arch.ce.nihon-u.ac.jp
}

(Received: November 16 ${ }^{\text {th }}, 2017$; Accepted: March $\left.4^{\text {th }}, 2018\right)$

\begin{abstract}
The use of the frequency-dependent spectral method in structural dynamic related problems is known to provide very accurate solutions while reducing the number of degree-of-freedom to resolve the computational and cost drawbacks. This paper investigated the vibrational characteristics of a rigid pavement road which is modeled by an isotropic Levy-type rectangular thin plates. The Spectral Element Method (SEM) in the frequency domain is developed to formulate the free vibration problems of the plate. Transcendental stiffness matrices are well established in vibration, derived from the exact analytical solutions of the differential equations of a plate element. The present spectral element model has four linetype degree-of-freedoms (DOF) on each edge of the Levy-type rectangular plate. Natural frequencies are found using the Wittrick-Williams algorithm. Numerical examples are given to show the effectiveness, efficiency, and accuracy of the SEM by using one element, unlike the FEM, the SEM gives exact solutions of the natural frequencies of plates without element discretization procedures.
\end{abstract}

Keywords: Free vibration; Frequency domain; Levy-type plate; Rigid pavement; Spectral Element Method

\section{INTRODUCTION}

Because the dynamic behavior and characteristics of a rigid pavement road are of great importance in civil engineering, it is necessary to predict them accurately in an efficient and economical technique. The Finite Element Method (FEM) is probably one of the dominant and common computation methods available in many fields of science and engineering. The vibration pattern of a rigid pavement road varies depending on the vibration frequency, and its wavelength at high frequency is very low. As a sufficiently accurate dynamic response can only be obtained by taking all necessary high-frequency wave modes, the size of meshes used in the finite element modeling must be sufficiently small, comparable to the lowest wavelength of the vibrating rigid pavement model. Since the conventional finite element models are formulated by using frequency independent polynomial shape functions, the FEM cannot accommodate all necessary highfrequency wave modes of interest without discretization. Thus the FEM solutions become inaccurate, especially at high frequencies, where associated wavelengths are very short. The socalled h-method is one of a well-known scheme for enhancing the FEM accuracy by refining the meshes. Unfortunately, this scheme will make a massive number of degree of freedoms (DOF), and hence from the computational aspect, the conventional FEM often becomes prohibitive for the most complex, rigid plate model.

An alternative scheme to improve the accuracy of the solution is to use the shape functions, which depend on the vibration frequency of the plate. Hence, the shape functions will be frequency dependent, and they are known as dynamic shape functions in the literature. As the dynamic shape functions can readily consider all necessary high-frequency wave modes of 
interest, exceptionally highly accurate solutions can be obtained, and the need to refine the meshes is no longer necessary. This sophisticated concept has directed to the so-called Dynamic Stiffness Method (DSM) [1-2]. As the exact dynamic stiffness matrix is formulated by using exact dynamic shape functions, it treats the mass distribution in a structure member implicitly. Thus only one single element is sufficient for a regular part of a rigid plate model, regardless of its length between any two successive structural or material discontinuities, to acquire exact solutions. That is, we no longer need to refine a regular part of a structure into multiple fine meshes. It will significantly reduce the size of the problem, in other words, the total number of meshes and DOF. In due course, this will significantly reduce the computation cost and time, together with improving the solution accuracy by reducing the computer round-off errors. Also, the DSM provides an infinite number of eigensolutions from the exact dynamic stiffness matrix represented in terms of a minimum number of DOF. Because the exact dynamic stiffness matrices are stiffness formulated (as the conventional finite element stiffness matrices are), they can be assembled in an analogous way to that used in the FEM. Thus, the meshing and assembly features of the FEM can be applied similarly to the DSM.

In Spectral Analysis Method (SAM) [3], the solutions to the governing differential equations are represented by the superposition of an infinite number of wave modes of different frequencies (or periods). The scheme related to the continuous Fourier transform of the solutions. This approach involves determining an infinite set of spectral components (or Fourier coefficients) in the frequency domain and performing the Inverse Fourier transform to reconstruct the time histories of the solutions. The continuous Fourier transform is feasible only when the function is mathematically simple. Thus, instead of using the continuous Fourier transform, the Discrete Fourier Transform (DFT) is widely used in practice.

Narayanan and Beskos [4] combined the features of the DSM with those of the SAM by introducing the fundamental concept of the Spectral Element Method (SEM). As illustrated in Fig. 1., the SEM can be considered as the combination of the main features of the conventional FEM, DSM and SAM. The key features of each method can be summarized as follows:

a Key features of FEM : Meshing (spatial discretization) and the assembly of finite elements.

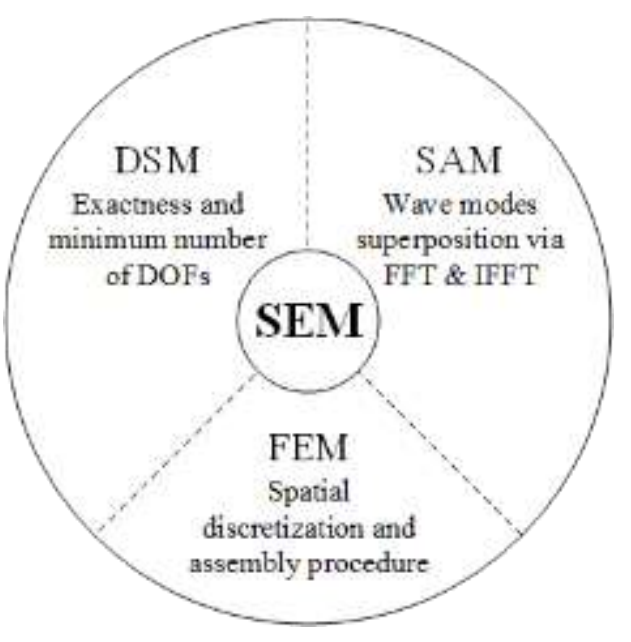

Fig. 1. Main features of the SEM

b Key features of DSM: Accuracy from the exact dynamic stiffness matrix formulated by a minimum number of DOF.

c Key features of SAM: Superposition of wave modes via DFT theory and FFT algorithm. 
In this study, the free vibrations of rectangular plates are analyzed. The Levy-type plate theory is based on the first order shear deformation plate theory and the governing equations which can be obtained from both the Maxwell equation and Hamilton principle. The exact natural frequencies of the free vibration plate are investigated. Comparison studies are performed to verify the validity of the SEM results. Moreover, the closed-form solutions of isotropic plate based on the Classical Plate Theory (CPT) [5-7] are also referred for the verification purpose. The effects of plate size aspect ratio on the natural frequencies of the isotropic Levy-type plates are studied and discussed.

\section{LEVY-TYPE PLATE}

The levy-type plate is defined by a rectangular plate with at least two parallel sides simply supported and arbitrary at the other edges boundary conditions. The governing equations yield to the coupled partial differential equations which can be decoupled by introducing auxiliary functions. The decoupled equations can be solved analytically by using the SEM.

\section{Equation of Motion of a Levy-type plate}

Figure 2. shows a rectangular Levy-type plate with two simply supported sides parallel to the $\mathrm{x}$-axis and the other two arbitrary boundary conditions at the opposite sides parallel to the $\mathrm{y}$-axis.

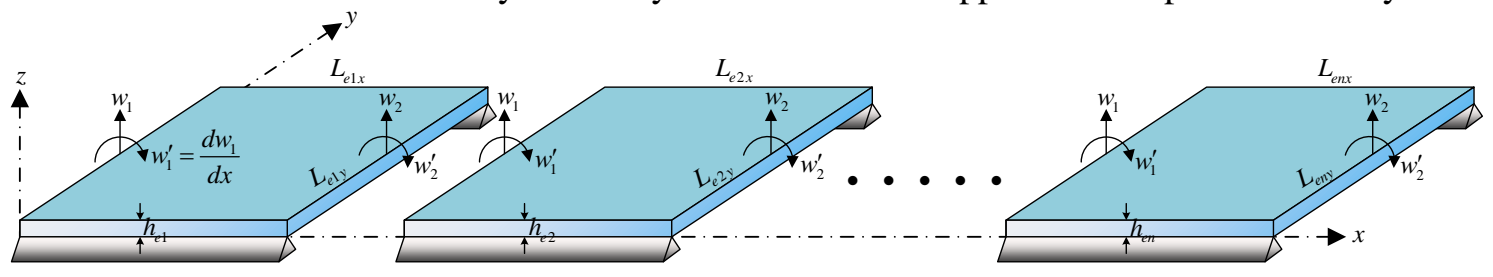

Fig. 2. Levy-type rectangular plate in the FEM scheme

The Levy-type plate has the size of Lx and Ly in the $\mathrm{x}$ - and $\mathrm{y}$ - directions, respectively. The homogeneous partial differential equations of small transverse vibration $w(x, y, t)$ of the plate is represented by the CPT,

$$
D\left(\frac{\partial^{4} w}{\partial x^{4}}+2 \frac{\partial^{4} w}{\partial x^{2} \partial y^{2}}+\frac{\partial^{4} w}{\partial y^{4}}\right)+\rho h \ddot{w}-\frac{\rho h^{3}}{12}\left(\frac{\partial^{2} \ddot{w}}{\partial x^{2}}+\frac{\partial^{2} \ddot{w}}{\partial y^{2}}\right)=0
$$

where, is the thinness, is the mass density and is the bending rigidity which is defined by $D=\frac{E h^{3}}{12\left(1-v^{3}\right)}, E$ is the elastic modulus and $v$ is the Poisson's ratio of the plate. The second and third terms in Eq. (1) stand for the translation and rotary inertia of the plate, respectively.

\section{Spectral Element Solution}

Assuming the dynamic response of the plate in n-domain spectral in the following form:

$$
\begin{aligned}
& w(x, y, t)=\frac{1}{N} \sum_{n=1}^{N} W_{n}\left(x, y ; \omega_{n}\right) e^{i \omega_{n} t} \\
& W_{n}\left(x, y ; \omega_{n}\right)=X_{n}(x) Y_{n}(y)
\end{aligned}
$$

Where $W_{n}\left(x, y ; \omega_{n}\right)$ is the spectral components of the $w(x, y, t), N$ is the sampling number and $\omega_{n}$ is the $n$-th natural frequency. The spectral component $W_{n}\left(x, y ; \omega_{n}\right)$ is obtained from multiplication of $X_{n}(x)$ and $Y_{n}(y)$ which are the displacement functions in $x$ - and $y$-directions, respectively. 
By assuming the $Y_{n}(y)$ solution in $\mathrm{m}$-domain spectral form as :

$$
Y_{n}\left(y ; k_{y n m}\right)=\frac{1}{M} \sum_{m=1}^{M} Y_{n m} e^{i k_{y n m} y}
$$

where, $M$ is the sampling number and

$$
k_{y n m}=\frac{m \pi}{L_{y}} \quad(m=1,2,3, \ldots)
$$

Substituting Eq. (4) into the spectral component of Eq. (3), results in

$$
\begin{aligned}
& W_{n}\left(x, y ; \omega_{n m}\right)=X_{n}(x) Y_{n}(y)=\frac{1}{M} \sum_{m=1}^{M} X_{n}\left(x ; k_{y n m}, \omega_{n m}\right) Y_{n m} e^{i k_{y m m} y} \\
& W_{n}\left(x, y ; \omega_{n}\right)=\frac{1}{M} \sum_{m=1}^{M} W_{n m}\left(x ; k_{y n m}, \omega_{n m}\right) e^{i k_{y n n} y}
\end{aligned}
$$

with,

$$
W_{n m}\left(x ; k_{y n m}, \omega_{n m}\right)=X_{n}\left(x ; k_{y n m}, \omega_{n m}\right) Y_{n m}
$$

Substituting Eq. (6) into Eq. (2), results in,

$$
\begin{aligned}
w(x, y, t) & =\frac{1}{N} \sum_{n=1}^{N} W_{n}\left(x, y ; \omega_{n}\right) e^{i \omega_{n} t} \\
& =\frac{1}{N} \sum_{n=1}^{N}\left(\frac{1}{M} \sum_{m=1}^{M} W_{n m}\left(x ; k_{y n m}, \omega_{n m}\right) e^{i k_{y n n} y}\right) e^{i \omega_{n n n} t}
\end{aligned}
$$

The derivations of $W_{n m}\left(x, y ; \omega_{n m}\right)$ at $n$ and $m$ in Eq. (8) with respect to $x, y$ and $t$ are given as,

$$
\begin{aligned}
& \frac{\partial w}{\partial x}=W_{n m}^{\prime}\left(x ; k_{y n m}, \omega_{n m}\right) e^{i k_{y n n} y} e^{i \omega_{n n} t} \quad \frac{\partial^{2} w}{\partial x^{2}}=W_{n m}^{\prime \prime}\left(x ; k_{y n m}, \omega_{n m}\right) e^{i k_{y n m} y} e^{i \omega_{n n} t} \\
& \frac{\partial^{4} w}{\partial x^{4}}=W_{n m}^{\prime \prime \prime}\left(x ; k_{y n m}, \omega_{n m}\right) e^{i k_{y m m} y} e^{i \omega_{n n} t} \quad \frac{\partial^{4} w}{\partial x^{2} \partial y^{2}}=\left(-k_{y n m}^{2}\right) W_{n m}^{\prime \prime}\left(x ; k_{y n m}, \omega_{n m}\right) e^{i k_{y m m} y} e^{i \omega_{n m} t} \\
& \frac{\partial^{4} w}{\partial y^{4}}=\left(k_{y n m}^{4}\right) W_{n m}\left(x ; k_{y n m}, \omega_{n m}\right) e^{i k_{y n n} y} e^{i \omega_{n m} t} \quad \frac{\partial^{2} w}{\partial t^{2}}=\left(-\omega_{n m}^{2}\right) W_{n m}\left(x ; k_{y n m}, \omega_{n m}\right) e^{i k_{y m m} y} e^{i \omega_{n n} t} \\
& \frac{\partial^{2} \ddot{w}}{\partial x^{2}}=\left(-\omega_{n m}^{2}\right) W_{n m}^{\prime \prime}\left(x ; k_{y n m}, \omega_{n m}\right) e^{i k_{y m n} y} e^{i \omega_{n m} t} \\
& \frac{\partial^{2} \ddot{w}}{\partial y^{2}}=\left(-\omega_{n m}^{2}\right)\left(-k_{y n m}^{2}\right) W_{n m}\left(x ; k_{y n m}, \omega_{n m}\right) e^{i k_{y n n} y} e^{i \omega_{n n} t}
\end{aligned}
$$

Herewith ()$^{\prime}=\frac{d()}{\partial x}$ its derivation operator with respect to the $x$-axis. Then, the homogeneous differential partial equation of Eq. (1) can be expressed as follow,

$$
D\left\{W_{n m}^{\prime \prime \prime}-2 k_{y n m}^{2} W_{n m}^{\prime \prime}+k_{y n m}^{4} W_{n m}\right\}-\omega_{n m}^{2} \rho h W_{n m}+\frac{\rho h^{3}}{12} \omega_{n m}^{2}\left(W_{n m}^{\prime \prime}-k_{y n m}^{2} W_{n m}\right)=0
$$


Assuming the spectral solution in the form

$$
W_{n m}\left(x ; k_{y n m}, \omega_{n m}\right)=W_{n m}=c_{n m} e^{-i k_{x n m} x}
$$

moreover, its derivatives as follow,

$$
W_{n m}^{\prime \prime}=\left(k_{x n m}^{2}\right) c_{n m} e^{-i k_{x x m} x}=-k_{x n m}^{2} W_{n m} \quad \text { and } \quad W_{n m}^{\prime \prime \prime \prime}=\left(k_{x n m}^{4}\right) c_{n m} e^{i k_{x n m} x}=k_{x n m}^{4} W_{n m} .
$$

Substituting Eq. (10) into Eq. (9), results in

$$
k_{x n m}^{4}+2 k_{x n m}^{2}\left(k_{y n m}^{2}-\omega_{n m}^{2} \frac{\rho h^{3}}{24 D}\right)+k_{y n m}^{4}-\omega_{n m}^{2} \frac{\rho h^{3}}{12 D} k_{y n m}^{2}-\omega_{n m}^{2} \frac{\rho h}{D}=0
$$

defining the translation and rotary inertia components as $A_{n}$ and $B_{n}$, respectively, as

$$
A_{n}^{2}=\omega_{n}^{2} \frac{\rho h}{D} \quad ; \quad B_{n}^{2}=\omega_{n}^{2} \frac{\rho h^{3}}{12 D}
$$

Equation (11) becomes,

$$
k_{x n m}^{4}+2 k_{x n m}^{2}\left(k_{y n m}^{2}-\frac{1}{2} B_{n}^{2}\right)+k_{y n m}^{4}-B_{n}^{2} k_{y n m}^{2}-A_{n}^{2}=0
$$

Four solutions wavenumbers $k_{x n m}$ of Eq. (12) are given by

$$
\begin{array}{ll}
k_{x n m 1}=-\frac{1}{2} \sqrt{2 B_{n}^{2}-4 k_{y n m}^{2}-2 \sqrt{4 A_{n}^{2}+B_{n}^{4}}} \quad, \quad k_{x n m 2}=\frac{1}{2} \sqrt{2 B_{n}^{2}-4 k_{y n m}^{2}-2 \sqrt{4 A_{n}^{2}+B_{n}^{4}}} \\
k_{x n m 3}=-\frac{1}{2} \sqrt{2 B_{n}^{2}-4 k_{y n m}^{2}+2 \sqrt{4 A_{n}^{2}+B_{n}^{4}}} \quad, \quad k_{x n m 4}=\frac{1}{2} \sqrt{2 B_{n}^{2}-4 k_{y n m}^{2}+2 \sqrt{4 A_{n}^{2}+B_{n}^{4}}}
\end{array}
$$

The general solution of Eq. (10) can be written as

$$
W_{n m}\left(x ; k_{y n m}, \omega_{n}\right)=c_{n m 1} e^{-i k_{x n m 1} x}+c_{n m 2} e^{-i k_{x n m 2} x}+c_{n m 3} e^{-i k_{x m m 3} x}+c_{n m 4} e^{-i k_{x m m} 4}
$$

alternatively, in matrix notation

$$
W_{n m}\left(x ; k_{y n m}, \omega_{n m}\right)=\mathbf{E}_{n m}\left(x ; k_{y n m}, \omega_{n m}\right) \mathbf{c}_{n m}
$$

where

$$
\begin{aligned}
\mathbf{E}_{n m}\left(x ; k_{y n m}, \omega_{n m}\right) & =\left[\begin{array}{llll}
e^{-i k_{x m m 1} x} & e^{-i k_{x n m 2} x} & e^{-i k_{x n m 3} x} & e^{-i k_{x n m} 4}
\end{array}\right] \\
\mathbf{c}_{n m}= & {\left[\begin{array}{llll}
c_{n m 1} & c_{n m 2} & c_{n m 3} & c_{n m 4}
\end{array}\right]^{T} }
\end{aligned}
$$

\section{Simply supported Levy-type plate}

Per definition, the Levy-type plate is defined by a rectangular plate with at least two parallel sides simply supported and arbitrary at the other edges boundary conditions. For a simply supported condition at all sides, the spectral nodal DOF with the boundary conditions at $x=0$ and $x=L x$ in the frequency $\omega$-domain is given as follow

$$
\mathbf{d}_{n}\left(\omega_{n}\right) \equiv\left\{\begin{array}{c}
W_{1 n}\left(y ; \omega_{n}\right) \\
\Theta_{1 n}\left(y ; \omega_{n}\right) \\
W_{2 n}\left(y ; \omega_{n}\right) \\
\Theta_{2 n}\left(y ; \omega_{n}\right)
\end{array}\right\}=\left\{\begin{array}{c}
W_{n}\left(0, y ; \omega_{n}\right) \\
W_{n}^{\prime}\left(0, y ; \omega_{n}\right) \\
W_{n}\left(L_{x}, y ; \omega_{n}\right) \\
W_{n}^{\prime}\left(L_{x}, y ; \omega_{n}\right)
\end{array}\right\}
$$


Representing the boundary conditions in spectral forms of the wavenumber $k_{y}$-domain as

$$
\left\{\begin{array}{l}
W_{1 n}\left(y ; \omega_{n}\right) \\
\Theta_{1 n}\left(y ; \omega_{n}\right) \\
W_{2 n}\left(y ; \omega_{n}\right) \\
\Theta_{2 n}\left(y ; \omega_{n}\right)
\end{array}\right\}=\frac{1}{M} \sum_{m=1}^{M}\left\{\begin{array}{c}
W_{1 n m} e^{-i k_{y m m} y} \\
\Theta_{1 n m} e^{-i k_{y m m} y} \\
W_{2 n m} e^{-i k_{y m m} y} \\
\Theta_{2 n m} e^{-i k_{y m m} y}
\end{array}\right\}
$$

By substituting Eqs. (6) and (17) into Eq. (16),

$$
\mathbf{d}_{n m}\left(k_{y n m}, \omega_{n m}\right) \equiv\left\{\begin{array}{l}
W_{1 n m}\left(k_{y n m}, \omega_{n m}\right) \\
\Theta_{1 n m}\left(k_{y n m}, \omega_{n m}\right) \\
W_{2 n m}\left(k_{y n m}, \omega_{n m}\right) \\
\Theta_{2 n m}\left(k_{y n m}, \omega_{n m}\right)
\end{array}\right\}=\left\{\begin{array}{c}
W_{n m}\left(0 ; k_{y n m}, \omega_{n m}\right) \\
W_{n m}^{\prime}\left(0 ; k_{y n m}, \omega_{n m}\right) \\
W_{n m}\left(L_{x} ; k_{y n m}, \omega_{n m}\right) \\
W_{n m}^{\prime}\left(L_{x} ; k_{y n m}, \omega_{n m}\right)
\end{array}\right\}
$$

where

$$
\boldsymbol{\Phi}_{n m}\left(k_{y n m}, \omega_{n m}\right)=\left[\begin{array}{cccc}
1 & 1 & 1 & 1 \\
-i k_{x n m 1} & -i k_{x n m 2} & -i k_{x n m 3} & -i k_{x n m 4} \\
e_{n m 1} & e_{n m 2} & e_{n m 3} & e_{n m 4} \\
-i k_{x n m 1} e_{n m 1} & -i k_{x n m 2} e_{n m 2} & -i k_{x n m 3} e_{n m 3} & -i k_{x n m 4} e_{n m 4}
\end{array}\right]
$$

with

$$
e_{n m j}=e^{-i k_{x m m j} L_{x}} \quad(j=1,2,3,4)
$$

By substituting Eq. (6) into the resultant bending moments and transverse shearing force at $x$ boundary condition,

$$
\begin{aligned}
& M_{x x n}\left(x, y ; \omega_{n m}\right)=D\left(\frac{\partial^{2} W_{n}}{\partial x^{2}}+v \frac{\partial^{2} W_{n}}{\partial y^{2}}\right) \\
& Q_{x z n}\left(x, y ; \omega_{n m}\right)=-D\left(\frac{\partial^{3} W_{n}}{\partial x^{3}}+(2-v) \frac{\partial^{3} W_{n}}{\partial x \partial y^{2}}\right)+\frac{\rho h^{3}}{12}\left(\frac{\partial \ddot{w}}{\partial x}\right)
\end{aligned}
$$

we can obtain,

$$
\begin{aligned}
& M_{x x n}\left(x, y ; \omega_{n m}\right)=\frac{1}{M} \sum_{m=1}^{M} M_{x x n m}\left(x ; k_{y n m}, \omega_{n m}\right) e^{-i k_{y n m} y} \\
& Q_{x z n}\left(x, y ; \omega_{n m}\right)=\frac{1}{M} \sum_{m=1}^{M} Q_{x z n m}\left(x ; k_{y n m}, \omega_{n m}\right) e^{-i k_{y m m} y}
\end{aligned}
$$

where

$$
\begin{aligned}
& M_{x x n m}\left(x ; k_{y n m}, \omega_{n m}\right)=D\left(W_{n m}^{\prime \prime}-v k_{y n m}^{2} W_{n m}\right) \\
& Q_{x z n m}\left(x ; k_{y n m}, \omega_{n m}\right)=-D\left[W_{n m}^{\prime \prime \prime}-(2-v) k_{y n m}^{2} W_{n m}^{\prime}\right]-\omega_{n m}^{2} \frac{\rho h^{3}}{12} W_{n m}^{\prime}
\end{aligned}
$$


Representing the spectral components (in the frequency $\omega$-domain) of the resultant transverse shearing force and bending moment as

$$
\mathbf{f}_{n}\left(\omega_{n}\right) \equiv\left\{\begin{array}{c}
Q_{1 n}\left(y ; \omega_{n}\right) \\
M_{1 n}\left(y ; \omega_{n}\right) \\
Q_{2 n}\left(y ; \omega_{n}\right) \\
M_{2 n}\left(y ; \omega_{n}\right)
\end{array}\right\}=\left\{\begin{array}{c}
-Q_{x z n}\left(0, y ; \omega_{n m}\right) \\
M_{x x n}\left(0, y ; \omega_{n m}\right) \\
-Q_{x z n}\left(L_{x}, y ; \omega_{n m}\right) \\
M_{x x n}\left(L_{x}, y ; \omega_{n m}\right)
\end{array}\right\}
$$

Represent the resultant transverse shear force and bending moment in the spectral forms in the wavenumber $k_{y}$-domain as

$$
\left\{\begin{array}{c}
Q_{1 n}\left(y ; \omega_{n}\right) \\
M_{1 n}\left(y ; \omega_{n}\right) \\
Q_{2 n}\left(y ; \omega_{n}\right) \\
M_{2 n}\left(y ; \omega_{n}\right)
\end{array}\right\}=\frac{1}{M} \sum_{m=1}^{M}\left\{\begin{array}{c}
Q_{1 n m} e^{-i k_{y m m} y} \\
M_{1 n m} e^{-i k_{y m m} y} \\
Q_{2 n m} e^{-i k_{y n m} y} \\
M_{2 n m} e^{-i k_{y m m} y}
\end{array}\right\}
$$

By substituting Eqs. (24) and (26) into Eq. (25), we can obtain

$$
\mathbf{f}_{n m}\left(k_{y n m}, \omega_{n m}\right) \equiv\left\{\begin{array}{c}
Q_{1 n m}\left(k_{y n m}, \omega_{n m}\right) \\
M_{1 n m}\left(k_{y n m}, \omega_{n m}\right) \\
Q_{2 n m}\left(k_{y n m}, \omega_{n m}\right) \\
M_{2 n m}\left(k_{y n m}, \omega_{n m}\right)
\end{array}\right\}=\left\{\begin{array}{c}
-Q_{x z n m}\left(0 ; k_{y n m}, \omega_{n m}\right) \\
M_{x x n m}\left(0 ; k_{y n m}, \omega_{n m}\right) \\
-Q_{x z n m}\left(L_{x} ; k_{y n m}, \omega_{n m}\right) \\
M_{x x n m}\left(L_{x} ; k_{y n m}, \omega_{n m}\right)
\end{array}\right\}
$$

Substitution of Eq. (15) into the right-hand side of Eq. (27) gives

$$
\begin{aligned}
& M_{x x n m}\left(x ; k_{y n m}, \omega_{n m}\right)=D\left[\mathbf{E}_{n m}^{\prime \prime}(x)-v k_{y n m}^{2} \mathbf{E}_{n m}(x)\right] \mathbf{c}_{n m} \\
& Q_{x z n m}\left(x ; k_{y n m}, \omega_{n m}\right)=-D\left[\mathbf{E}_{n m}^{\prime \prime \prime}(x)-(2-v) k_{y n m}^{2} \mathbf{E}_{n m}^{\prime}(x)\right] \mathbf{c}_{n m}-\omega_{n m}^{2} \frac{\rho h^{3}}{12} \mathbf{E}_{n m}^{\prime}(x) \mathbf{c}_{n m}
\end{aligned}
$$

Applying Eq. (28) on the right-hand side of Eq. (27) gives

$$
\mathbf{f}_{n m}\left(k_{y n m}, \omega_{n m}\right)=D\left[\begin{array}{c}
\mathbf{E}_{n m}^{\prime \prime \prime}(0)-(2-v) k_{y n m}^{2} \mathbf{E}_{n m}^{\prime}(0)-B_{n}^{2} \mathbf{E}_{n m}^{\prime}(0) \\
\mathbf{E}_{n m}^{\prime \prime}(0)-v k_{y n m}^{2} \mathbf{E}_{n m}(0) \\
\mathbf{E}_{n m}^{\prime \prime \prime}\left(L_{x}\right)-(2-v) k_{y n m}^{2} \mathbf{E}_{n m}^{\prime}\left(L_{x}\right)-B_{n}^{2} \mathbf{E}_{n m}^{\prime}\left(L_{x}\right) \\
\mathbf{E}_{n m}^{\prime \prime}\left(L_{x}\right)-v k_{y n m}^{2} \mathbf{E}_{n m}\left(L_{x}\right)
\end{array}\right] \mathbf{c}_{n m}
$$

By using Eq. (15), the Eq. (29) can be written as

$$
\mathbf{f}_{n m}\left(k_{y n m}, \omega_{n m}\right)=\mathbf{G}_{n m}\left(k_{y n m}, \omega_{n m}\right) \mathbf{c}_{n m}
$$

where 


$$
\begin{aligned}
& \mathbf{G}_{n m}\left(k_{y n m}, \omega_{n m}\right)= \\
& D\left[\begin{array}{cc}
i k_{x n m 1}\left[k_{x n m 1}^{2}+(2-v) k_{y n m}^{2}+B_{n}^{2}\right] & i k_{x n m 2}\left[k_{x n m 2}^{2}+(2-v) k_{y n m}^{2}+B_{n}^{2}\right] \\
-\left(k_{x n m 1}^{2}+v k_{y n m}^{2}\right) & -\left(k_{x n m 2}^{2}+v k_{y n m}^{2}\right) \\
i k_{x n m 1}\left[k_{x n m 1}^{2}+(2-v) k_{y n m}^{2}+B_{n}^{2}\right] e_{n m 1} & i k_{x n m 2}\left[k_{x n m 2}^{2}+(2-v) k_{y n m}^{2}+B_{n}^{2}\right] e_{n m 2} \\
-\left(k_{x n m 1}^{2}+v k_{y n m}^{2}\right) e_{n m 1} & -\left(k_{x n m 2}^{2}+v k_{y n m}^{2}\right) e_{n m 2} \\
i k_{x n m 3}\left[k_{x n m 3}^{2}+(2-v) k_{y n m}^{2}+B_{n}^{2}\right] & i k_{x n m 4}\left[k_{x n m 4}^{2}+(2-v) k_{y n m}^{2}+B_{n}^{2}\right] \\
-\left(k_{x n m 3}^{2}+v k_{y n m}^{2}\right) & -\left(k_{x n m 4}^{2}+v k_{y n m}^{2}\right) \\
\ldots & i k_{x n m 3}\left[k_{x n m 3}^{2}+(2-v) k_{y n m}^{2}+B_{n}^{2}\right] e_{n m 3} \\
-\left(k_{x n m 3}^{2}+v k_{y n m}^{2}\right) e_{n m 3} & i k_{x n m 4}\left[k_{x n m 4}^{2}+(2-v) k_{y n m}^{2}+B_{n}^{2}\right] e_{n m 4} \\
& -\left(k_{x n m 4}^{2}+v k_{y n m}^{2}\right) e_{n m 4}
\end{array}\right]
\end{aligned}
$$

The constant vector $\mathbf{c}_{n m}$ can be eliminated from Eq. (30) by using Eq. (19) to obtain the spectral element equation for the Levy-type plate element as

$$
\begin{aligned}
\mathbf{f}_{n m}\left(k_{y n m}, \omega_{n m}\right) & =\mathbf{G}_{n m}\left(k_{y n m}, \omega_{n m}\right) \mathbf{c}_{n m} \\
= & \mathbf{G}_{n m}\left(k_{y n m}, \omega_{n m}\right) \mathbf{\Phi}_{n m}^{-1}\left(k_{y n m}, \omega_{n m}\right) \mathbf{d}_{n m}\left(k_{y n m}, \omega_{n m}\right) \\
= & \mathbf{S}\left(k_{y n m}, \omega_{n m}\right) \mathbf{d}_{n m}\left(k_{y n m}, \omega_{n m}\right)
\end{aligned}
$$

Where

$$
\mathbf{S}\left(k_{y n m}, \omega_{n m}\right)=\mathbf{G}_{n m}\left(k_{y n m}, \omega_{n m}\right) \mathbf{\Phi}_{n m}^{-1}\left(k_{y n m}, \omega_{n m}\right)
$$

The size of the matrix $\mathbf{S}\left(k_{y n m}, \omega_{n m}\right)$ is $4 \times 4$ that is the function of natural frequency $\omega_{n m}$ and wavenumber $k_{y n m}$. In a similar way to the FEM, this spectral element matrix of the Levy-type plate element can be discretized and assembled in the $x$-direction to form a global stiffness matrix system.

\section{LEVY-TYPE PLATE}

\section{Free Vibration}

Consider the geometry of two different aspect ratio of width and length of Levy-type plates as shown in Fig. 3. The following isotropic material properties of the plate are used in the calculation: elastic modulus $E=1$; Poisson's ratio $v=0.3$; and mass density $\rho=1.0$. The shear modulus is computed from $G=\frac{E}{2(1+v)}$. The plates are restrained at both opposite parallel sides as simply supported boundary conditions.
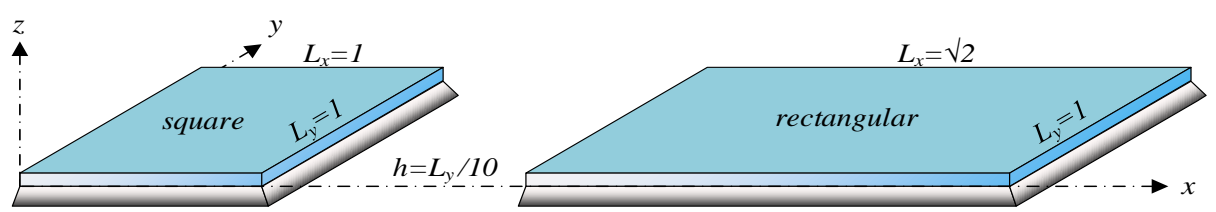

Fig. 3. Levy-type rectangular plate in the FEM scheme 
CPT with rotary inertia, $B_{n} \neq 0$

CPT without rotary inertia, $B_{n}=0$

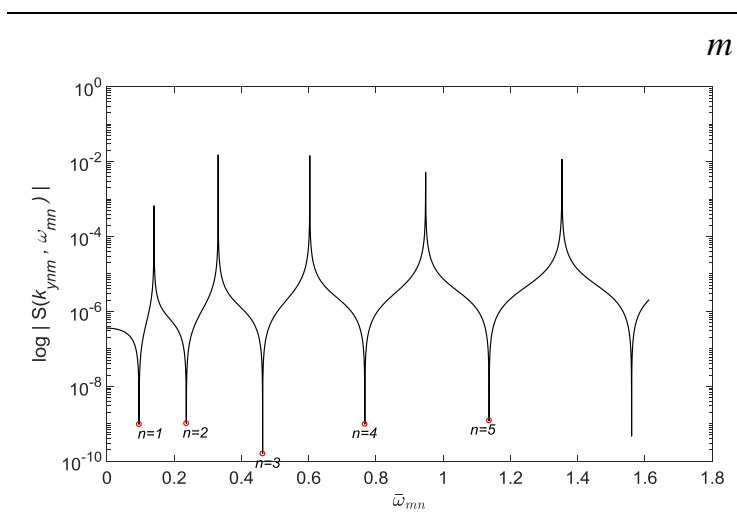

$m=1$

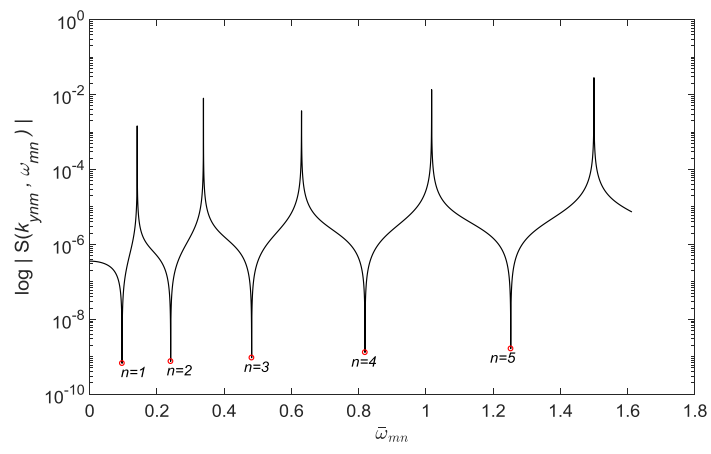

$m=2$
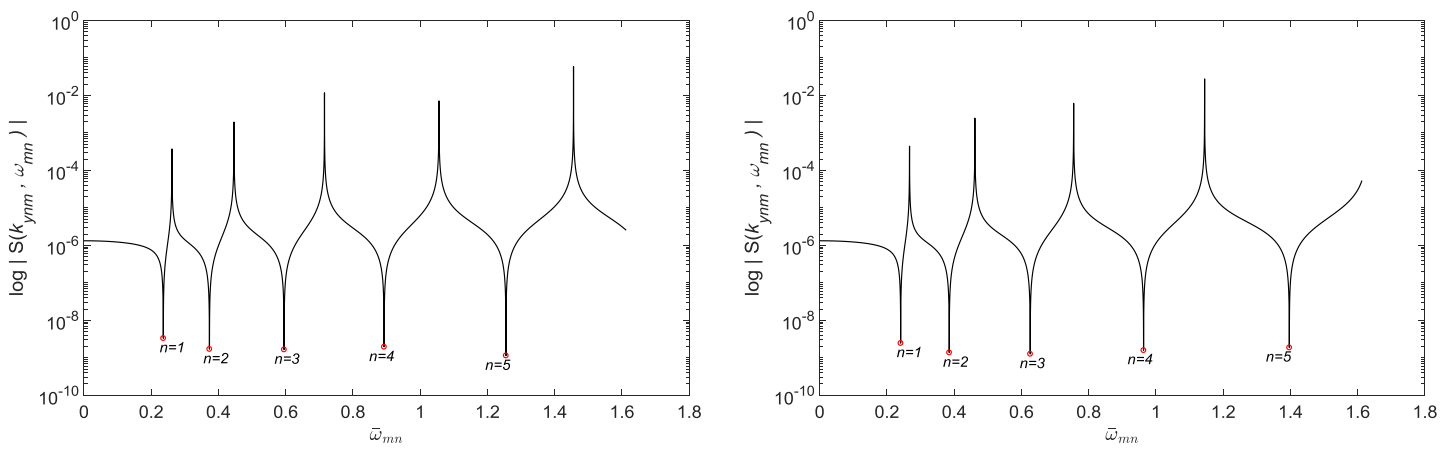

$m=3$
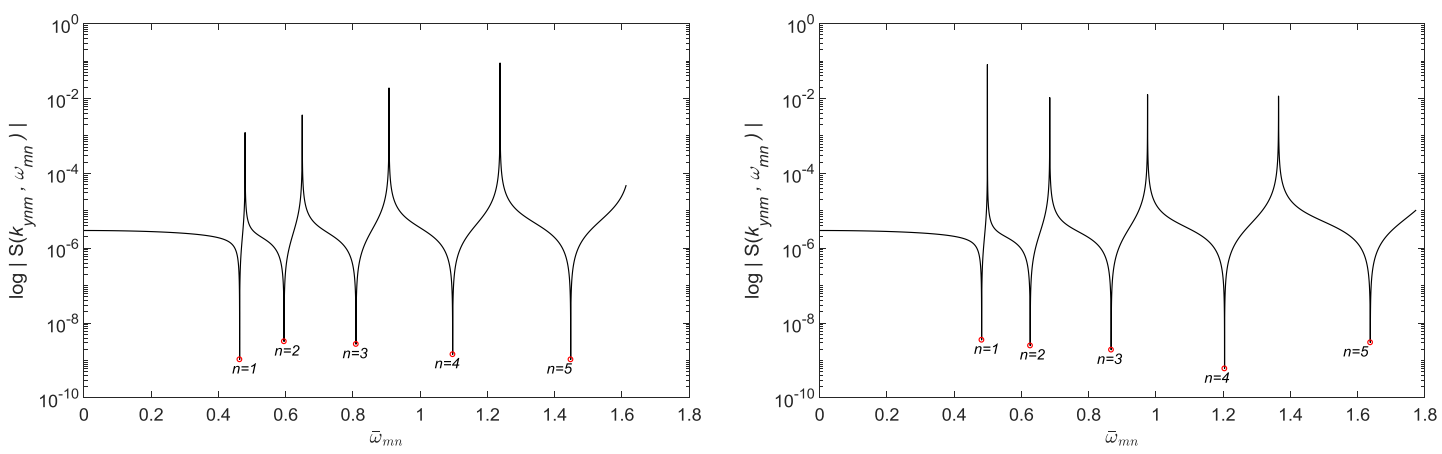

$m=4$
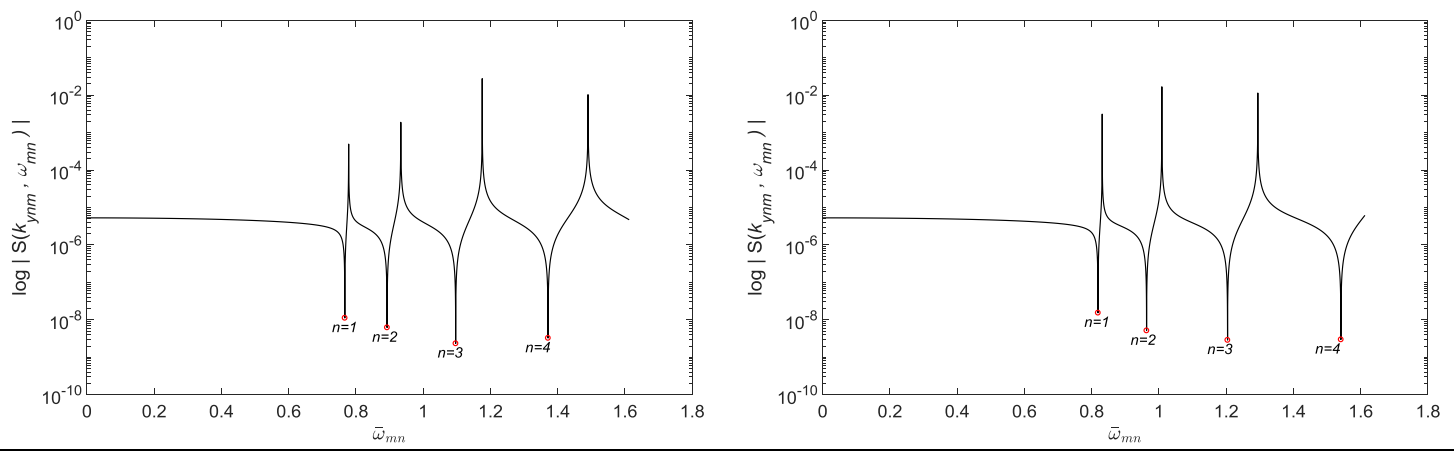

Fig. 4. SEM computed normalized natural frequencies, $\bar{\omega}_{m n}=\omega_{m n} h \sqrt{\rho / G}$ of a square plate $\left(L_{x} / L_{y}=1, L_{y} / h=10\right)$ 


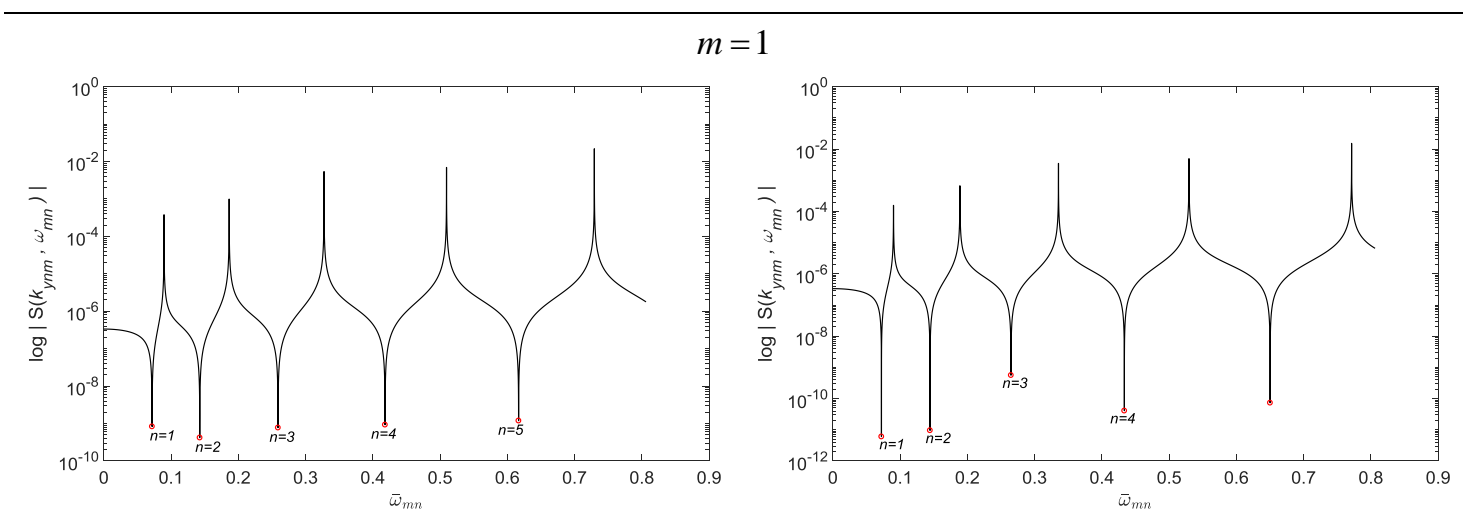

$m=2$
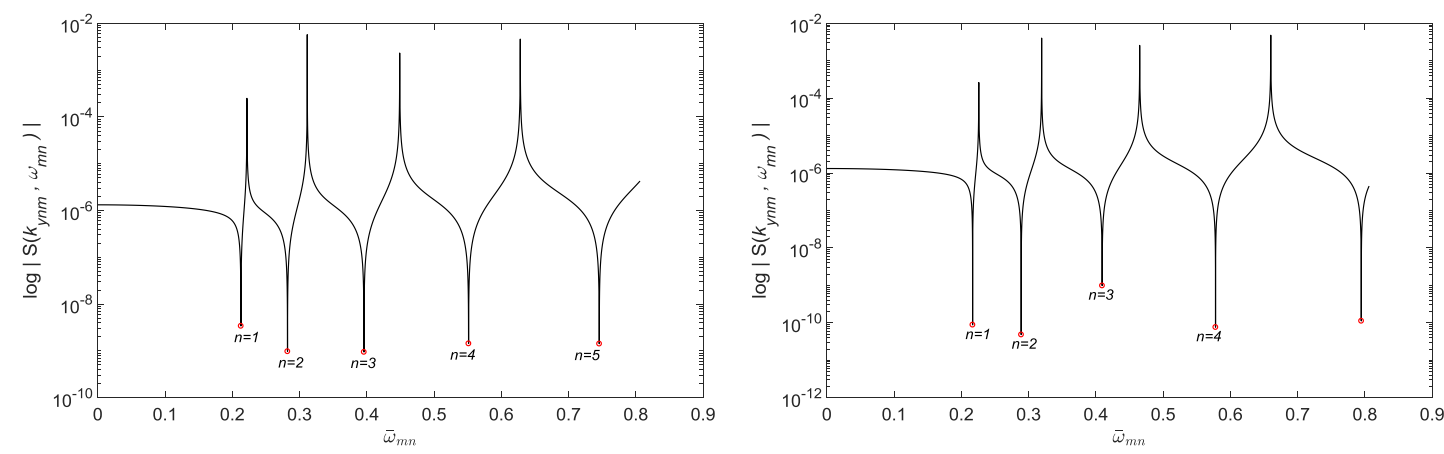

$m=3$
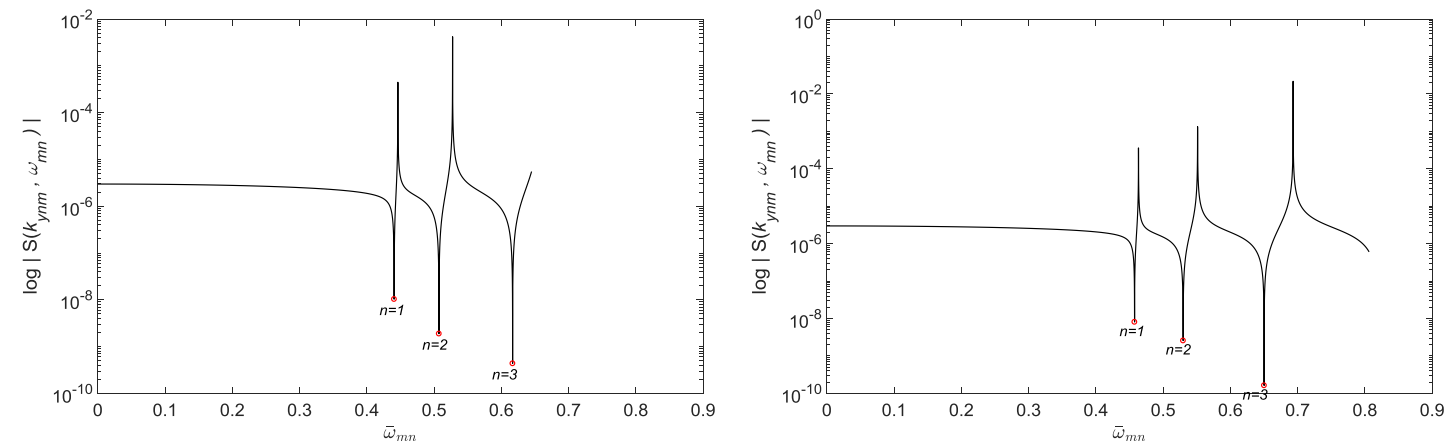

Fig. 5. SEM computed normalized natural frequencies, $\bar{\omega}_{m n}=\omega_{m n} h \sqrt{\rho / G}$ of a rectangular plate

$$
\left(L_{x} / L_{y}=\sqrt{2}, L_{y} / h=10\right)
$$

The natural frequencies of the plates are obtained from solving the eigenvalue problem of the spectral element model which can be reduced from Eg. (32) to be

$$
\mathbf{f}_{n m}\left(k_{y n m}, \omega_{n m}\right)=\mathbf{S}\left(k_{y n m}, \omega_{n m}\right) \mathbf{d}_{n m}\left(k_{y n m}, \omega_{n m}\right)=0
$$

The eigenfrequencies $\omega_{n m}(m=1,2,3 \ldots ; n=1,2,3 \ldots)$ are determined by using the condition that the determinant of $\mathbf{S}\left(k_{y n m}, \omega_{n m}\right)$ is zero at $\omega=\omega_{n m}$, that is,

$$
\left|\mathbf{S}\left(k_{y n m}, \omega_{n m}\right)\right|=0
$$


The Wittrick-Williams algorithm [8] is implemented to calculate the natural frequencies of the plates. The results of normalized natural frequencies of the plates are compared with the CPT solutions [5-7] in Figs. 4-5 and Tables 1-2 for with or without taking into account the rotary inertia.

Table 1. Comparison of normalized natural frequencies, $\bar{\omega}_{m n}=\omega_{m n} h \sqrt{\rho / G}$ of a square plate

$$
\left(L_{x} / L_{y}=1, L_{y} / h=10\right)
$$

\begin{tabular}{cccccc}
\hline & & \multicolumn{2}{c}{ With rotary inertia, $B_{n} \neq 0$} & \multicolumn{2}{c}{ Without rotary inertia, } \\
\cline { 3 - 6 } & $\mathbf{n}$ & SEM & CPT[5-6] & SEM \\
\cline { 3 - 6 } & & CPT[5-6] & 0.0955 & 0.0963 & 0.0963 \\
\hline 1 & 1 & 0.0955 & 0.2360 & 0.2408 & 0.2408 \\
\hline 1 & 2 & 0.2360 & 0.3732 & 0.3853 & 0.3853 \\
\hline 2 & 2 & 0.3732 & 0.4629 & 0.4816 & 0.4816 \\
\hline 1 & 3 & 0.4629 & 0.5951 & 0.6261 & 0.6261 \\
\hline 2 & 3 & 0.5951 & 0.5951 & 0.8187 & 0.8187 \\
\hline 1 & 4 & 0.7668 & 0.7668 & 0.8669 & 0.8669 \\
\hline 3 & 3 & 0.8090 & 0.8090 & 0.9632 & 0.9632 \\
\hline 2 & 4 & 0.8926 & 0.8926 & 1.2040 & 1.2040 \\
\hline 3 & 4 & 1.0965 & 1.0965 & 1.2521 & 1.2521 \\
\hline 1 & 5 & 1.1365 & 1.1365 & 1.3966 & 1.3966 \\
\hline 2 & 5 & 1.2549 & 1.2549 & 1.5411 & 1.5411 \\
\hline 4 & 4 & 1.3716 & 1.3712 & 1.6374 & 1.6374 \\
\hline 3 & 5 & 1.4475 & 1.4475 & &
\end{tabular}

Table 2. Comparison of normalized natural frequencies, $\bar{\omega}_{m n}=\omega_{m n} h \sqrt{\rho / G}$ of a rectangle plate

$$
\left(L_{x} / L_{y}=\sqrt{2}, L_{y} / h=10\right)
$$

\begin{tabular}{cccccc}
\hline & & \multicolumn{2}{c}{ With rotary inertia, $B_{n} \neq 0$} & \multicolumn{2}{c}{ Without rotary inertia, } \\
$B_{n}=0$
\end{tabular}




\begin{tabular}{cccccc}
\hline & & \multicolumn{2}{c}{ With rotary inertia, $B_{n} \neq 0$} & \multicolumn{2}{c}{ Without rotary inertia, } \\
$B_{n}=0$
\end{tabular}

\section{DISCUSSION AND RESULTS}

Numerical results from the CPT and SEM are depicted in Figs. 4-5 and tabulated in Tables 1-2. The results obtained using the SEM are found to give excellent exact values compared with the CPT values reported. Numerical examples are given to show the effectiveness, efficiency, and accuracy of the SEM by using only one element, unlike the FEM, the SEM gives exact solutions of the natural frequencies of plates without element discretization procedures.

The figures present the results of consecutive natural frequencies of $m$ and its corresponding $n$ modes of both plates with and without taking into account the rotary inertia. By taking into account the rotary inertia term in the plate equation, higher natural frequencies are obtained regardless of the vibration modes.

The SEM has shown that the transcendental stiffness matrices which are well established in free vibration problems can be derived from the exact analytical solutions of the differential equations of a plate element. The effectiveness of the Wittrick-Williams algorithm also has been proved suitable for use in the SEM formulation, as opposed to the approximation method by using the FEM.

Depends on the fundamental theory and concept of the plate element being considered, the SEM can be established accordingly to give the exact solution by using the least number of element.

\section{REFERENCES}

[1] Banerjee, J.R. (1977). Dynamic Stiffness Formulation for Structural Elements: A General Approach. Computers \& Structures, 63(1), 101-103.

[2] Leung, A.Y.T. (1993). Dynamic Stiffness and Substructures, London: Springer-Verlag.

[3] Doyle, J.F. (1997), Wave Propagation in Structures: Spectral Analysis Using Fast Discrete Fourier Transforms, New York: Springer.

[4] Narayanan, G.V. and Beskos, D.E. (1978). Use of Dynamic Influence Coefficients in Forced Vibration Problems with the aid of Fast Fourier transform. Computers \& Structures, 9(2), 145-150.

[5] Reddy, G.V. and Phan, N.D. (1985). Stability and Vibration of Isotropic, Orthotropic and Laminated Plates According to a Higher-Order Shear Deformation Theory. Journal of Sound and Vibration, 98(2), 157-170.

[6] Shimpi, R.P. and Patel, H.G. (2006). Free Vibrations of Plate using Two Variable Refined Plate Theory. Journal of Sound and Vibration, 296(4-5), 977-999.

[7] Wang, C.M., Reddy, J.N. and Lee, K.H. (2000), Shear Deformable Beams and Plates: Relationships with Classical Solutions, Oxford: Elsevier Science Ltd.

[8] Wittrick, W.H. and Williams, F.W. (1971), A General Algorithm for Computing Natural Frequencies of Elastic Structures. Quarterly Journal of Mechanics and Applied Mathematics, 24(3), 263-284. 